\title{
STROKE AND STROKE MIMICS: A CASE OF HIGH-GRADE GLIOMA
}

Clin Biomed Res. 2014;34(4):410-411 1 Serviço de Radiologia, Hospital de Clínicas de Porto Alegre. Porto Alegre, RS, Brazil.

Corresponding author: Juliana Ávila Duarte E-mail: juavila2003@yahoo.com.br Serviço de Radiologia Hospital de Clínicas de Porto Alegre Rua Ramiro Barcelos, 2350. 90035-903, Porto Alegre, RS, Brazil.

\section{Roberta Wolffenbüttel Argenti ${ }^{1}$, Juliana Avila Duarte ${ }^{1}$, Amália Izaura Nair Medeiros Klaes ${ }^{1}$, Francine Hehn Oliveira ${ }^{1}$, Mariangela Gheller Friedrich ${ }^{1}$, Lillian Gonçalves Campos ${ }^{1}$, Juliano Adams Perez ${ }^{1}$, Apio Claudio Antunes ${ }^{1}$, Sheila Ouriques Martins ${ }^{1}$, Leonardo Modesti Vedolin ${ }^{1}$}

The clinical diagnosis of acute stroke is inaccurate approximately 10\%$30 \%$ of the time, which can lead to unnecessary administration of thrombolytic therapy or delays in appropriate therapy ${ }^{1}$. Rapid and accurate neuroimaging triage is essential to guide therapy and exclude mimics. Although many conditions that mimic stroke clinically have imaging appearances that can overlap acute stroke, these conditions can be differentiated in most cases by using a careful pattern-based approach ${ }^{2}$.

We describe a case of 67 year-old male patient who had a clinic of wakeup stroke and whose first magnetic resonance image (MRI) an acute stroke of middle cerebral artery was diagnosed (figure $1 \mathrm{a}$ and $\mathrm{b}$ ). The patient did not improve, and a second MRI revealed a twofold growth of the lesion, with MRI findings compatible with tumor (figure $2 \mathrm{a}, \mathrm{b}$ and $\mathrm{c}$ ).

At surgery, an infiltrative lesion was found, and the anatomopathological exam showed that it was a high-grade glioma.

The diagnosis of ischemic stroke is often straight forward; however, the clinical diagnosis of acute stroke is inaccurate in many cases because many conditions, such as encephalitis, mass lesions, seizures, hypoglycemia, transient global amnesia, demyelinating disease, drug toxicity, and metabolic disturbances, have imaging appearances that can mimic acute or subacute infarction. Nonetheless, an accurate diagnosis can often be made by using a pattern-based approach ${ }^{3-5}$.
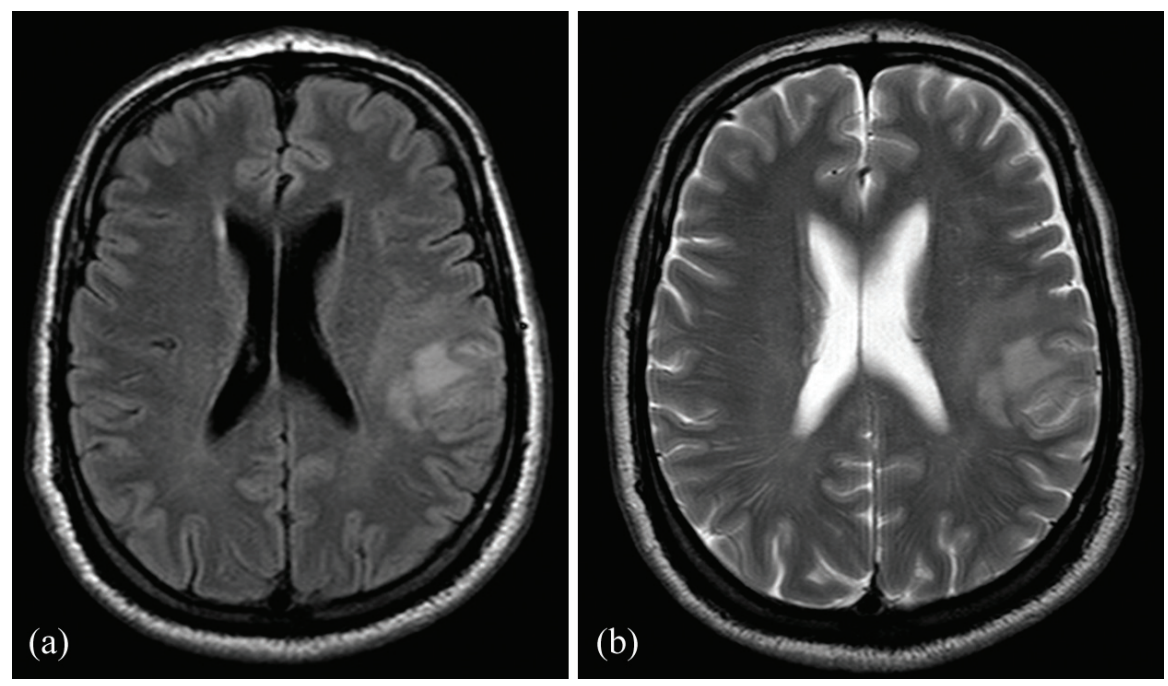

Figure 1: a - Axial flair weighted imaging - Hyperintense lesion on left temporal lobe with restricted diffusion on diffusion-weighted imaging (DWI) (DWI not shown here); $\mathrm{b}$ - Axial T2WI- Hyperintense lesion on left temporal lobe with restricted diffusion on DWI (DWI not shown here). 

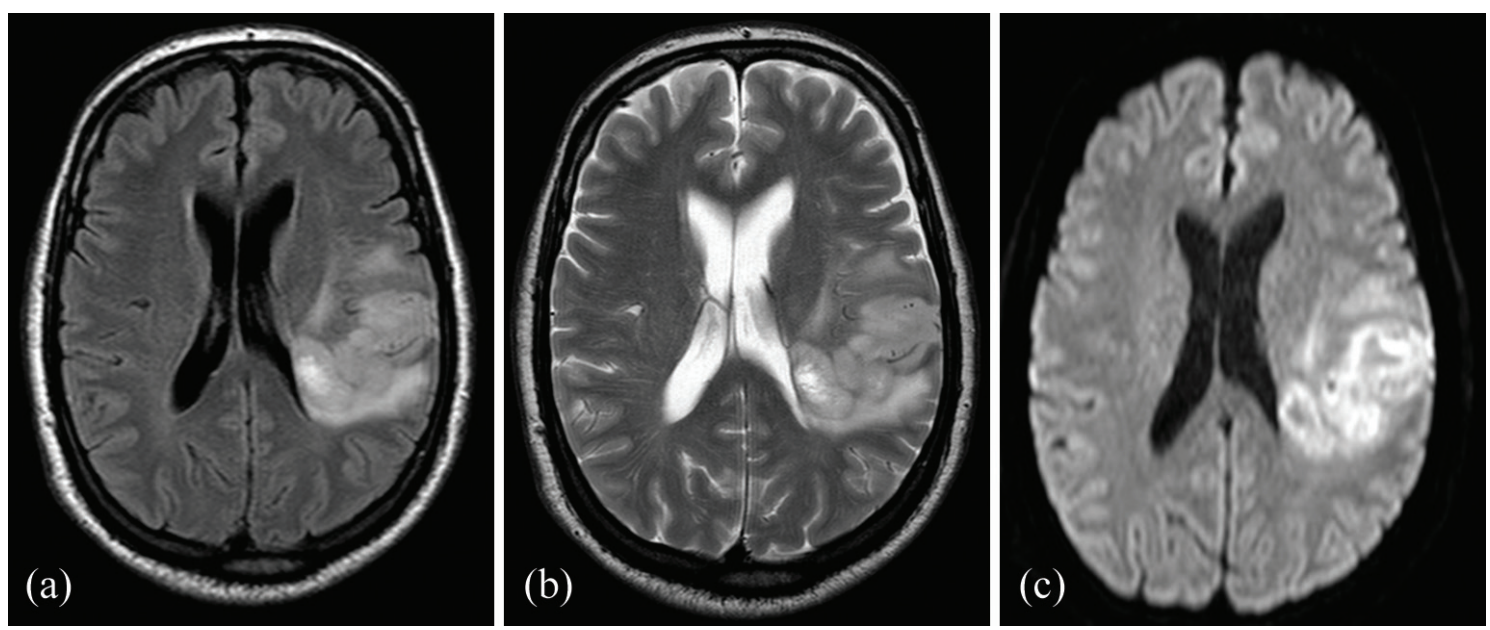

Figure 2: a - Axial flair weighted imaging - Enlargement of the hyperintense lesion on left temporal lobe with restricted diffusion on diffusion-weighted imaging (DWI); b - Axial T2WI - Hyperintense lesion on left temporal lobe with restricted diffusion on DWI; C - Axial DWI - Hyperintense lesion on left temporal lobe with restricted diffusion on DWI and apparent diffusion coefficient (ADC) map.

Patients presenting with focal neurological symptoms may suffer from ischemic stroke, intracerebral hemorrhage, or stroke mimics. Such patients are usually screened with a noncontrast-enhanced computed tomography to rule out hemorrhage and to detect early signs of ischemia. However, the sensitivity of noncontrast- enhanced computed tomography for acute stroke is far inferior to that of diffusion-weighted MRI and the latter is also very sensitive for identifying acute intracerebral hemorrhage. Most centers perform a MRI stroke protocol that takes long to accomplish and may therefore delay therapy ${ }^{6}$.

\section{REFERENCES}

1. Boulter DJ, Schaefer PW. Stroke and stroke mimics: a pattern-based approach. Semin Roentgenol. 2014;49(1):22-38. http://dx.doi. org/10.1053/j.ro.2013.10.005 PMid:24342673

2. Liu X, Almast J, Ekholm S. Lesions masquerading as acute stroke. $J$ Magn Reson Imaging. 2013;37(1):1534. http://dx.doi.org/10.1002/ jmri.23647. PMid:23255413

3. Kose A, Inal T, Armagan E, Kıyak $\mathrm{R}$, Demir $A B$. Conditions that mimic stroke in elderly patients admitted to the emergency department. $J$ Stroke Cerebrovasc Dis. 2013;22(8):e5227. http://dx.doi.org/10.1016/j. jstrokecerebrovasdis.2013.05.029. PMid:23830957

4. Magauran BG JR, Nitka M. Stroke mimics. Emerg Med Clin North Am. 2012;30(3):795-804. http://dx.doi. org/10.1016/j.emc.2012.06.006. PMid:22974649

5. Libman RB, Wirkowski E, Alvir J, Rao TH. Conditions that mimic stroke in the emergency department. Implications for acute stroke trials. Arch Neurol. 1995;52(11):111922. http://dx.doi.org/10.1001/ archneur.1995.00540350113023. PMid:7487564

6. Leker RR, Keigler G, Eichel R, Ben Hur T, Gomori JM, Cohen JE. Should DWI MRI be the primary screening test for stroke? Int J Stroke. 2014;9(6):696-7. http://dx.doi. org/10.1111/ijs.12316. PMid:25043518 